\title{
The Aftermaths of the SMC/LMC Encounter
}

\author{
Serge Demers \\ Département de Physique, Université de Montréal, Montreal Qc, \\ Canada H3C $3 \mathrm{~J} 7$ \\ W. E. Kunkel \\ Las Campanas Observatory, Carnegie Institution of Washington, La \\ Serera, Chile
}

\begin{abstract}
We present structural and kinematic evidence pointing to a gravitational interaction between the two Magellanic Clouds. We discuss, in particular, the results of our kinematic survey of carbon stars in the periphery of the LMC. Stars with peculiar velocities, in the LMC southwest, are identified as part of debris left on the SMC orbit around the LMC.
\end{abstract}

\section{Introduction}

The determination of the proper motion of the SMC by Irwin et al. (1996) implies that it is most certainly bound to the LMC and that it is currently moving away from it. The close encounter presumed to have taken place some $200 \mathrm{Myr}$ ago is once more supported by observations. The analysis of the radial velocities of nearly 1000 carbon stars (Kunkel et al. 1997a) in the periphery of both Clouds brings new facts, not only about the mass of the LMC (Kunkel et al. 1997b) but also about the geometry of the interaction. Some topics of this analysis are discussed here.

\section{The Inter-cloud Young Population}

Young star aggregates, extending east of the SMC wing, were discovered by Irwin et al. (1990). These stellar groups were first investigated by Grondin et al. (1992) and more recently by Demers \& Battinelli (1998) who showed that the distance to the five major aggregates decreases from the SMC side to the LMC side. They are indeed part of a "bridge" linking the two Clouds. Furthermore, the stellar population making up these aggregates was found to be very young: 10 to $25 \mathrm{Myr}$. This implies that some delayed star formation mechanisms must be at work in this region, if we believe that the tidal impact took place $\sim 200$ Myr ago.

The kinematics of the young inter-Cloud population is still barely known. It is, for example, not known if these stars are all part of a tidal bridge or if some of them are in the tidal tail which, according to interaction models, would 
be, seen from earth, behind the bridge. Clues concerning this come from the radial velocity of the two brightest stars in aggregate ICA 76 - the eastern most one, $8^{\circ}$ from the LMC center, which differs by $80 \mathrm{~km} \mathrm{~s}^{-1}$ (Demers et al. 1991). We are dealing here with main sequence stars of $\mathrm{V}>17$ thus requiring a large telescope to obtain spectra.

The further extension of the young inter-Cloud population toward the LMC has recently been investigated by Battinelli \& Demers (1999) who showed that blue stars are present in the western periphery of the $\mathrm{LMC}, 6^{\circ}$ from its center, but no stars as young as the ones of the inter-Cloud aggregates are seen. The origin of this patch of blue stars is not currently known.

\section{The Carbon Stars}

In order to increase substantially the number of kinematic probes we directed our interest toward carbon stars. Such red stars are easy to identify from color magnitude diagrams but they require spectra to confirm their nature. Carbon stars are more numerous and brighter than inter-Cloud upper main sequence stars. Furthermore, they are much more numerous than clusters to define the kinematics at large distance from the center of the LMC or the SMC. They are known to be of intermediate age, which means that they did not witness the first stages of the LMC or SMC formation. The few hundred carbon stars observed by us in the LMC have a velocity dispersion, (taking into account the translation and the rotation of the LMC), of $16 \mathrm{~km} \mathrm{~s}^{-1}$ which compared to the velocity dispersion of LPV (Hughes et al. 1991) must be 1 to 2 Gyr old, old enough to have experienced the last encounter.

As explained by Kunkel et al. (1997a), the red candidates for spectroscipic follow-ups are selected from UKST plate pairs scanned by the APM. As we reach smaller radial distances from the center of the LMC, the number of candidates becomes quite high. For this reason, in the inner zone, only very red stars were spectroscopically observed. Our survey of carbon stars is complete in the LMC periphery outside a deprojected radius of $6.2^{\circ}$. For the deprojection, we adopt the $\mathrm{HI}$ rotation center for the LMC center, an inclination of $33^{\circ}$ and a line of nodes at $168^{\circ}$.

As we can see from Figure 1, the distributions of the LMC carbon stars and of clusters are quite different in the periphery. The carbon stars extend to larger distances, one in the north east is as far as $20^{\circ}$ from its center. There are few clusters outside of the $6.2^{\circ}$ circle while there are many carbon stars. The reason for this different distribution is not known. Tidal interaction may have more facility to redistribute individual stars than clusters. It is also obvious that the cluster and carbon star distributions are asymmetric relative to the HI rotation center. The distributions are displaced toward the east.

\section{Radial Velocities}

Carbon stars as well as clusters, LPVs and HI can be used to investigate the orbital velocity of LMC members as a function of their radial distance. This has been described by Kunkel et al. (1997b) who used the data to estimate the LMC mass. Figure 2 presents the orbital velocities of the above mentioned probes for 
two annulii centered on the LMC. These velocities are corrected for the solar motions and for the translation motion of the LMC. Panel a) corresponds to the 4 to 20 degree zone and b) represents the 3 to 5 degree zone. One can easily see, in the upper panel, the points near PA $=220$ which do not follow the sinusoidal curve. These are, mostly hydrogen, in the inter-Cloud area. In the inner zone, displayed on panel b), one can identify points which do not follow the rotation. Carbon stars are among the points which "do not belong".
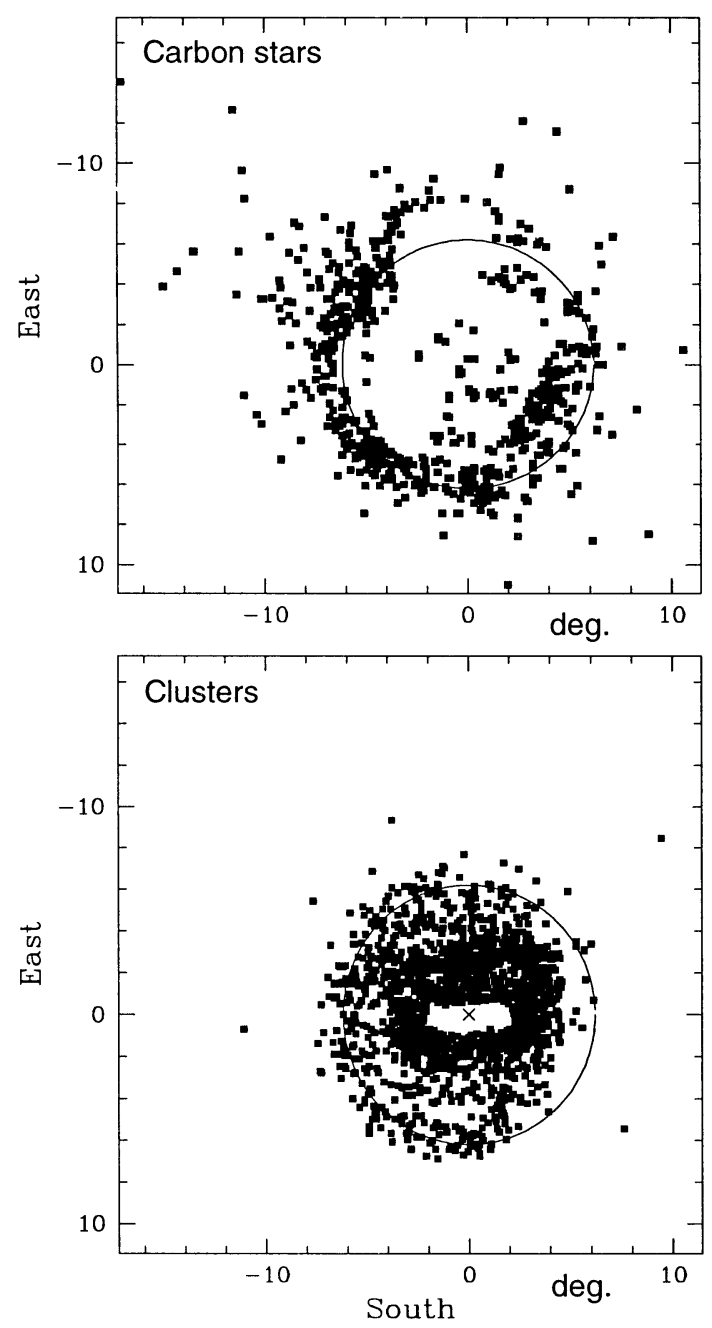

Figure 1. Distribution of carbon stars and clusters on the deprojected plane of the LMC. The circle has a radius of $6.2^{\circ}$. 


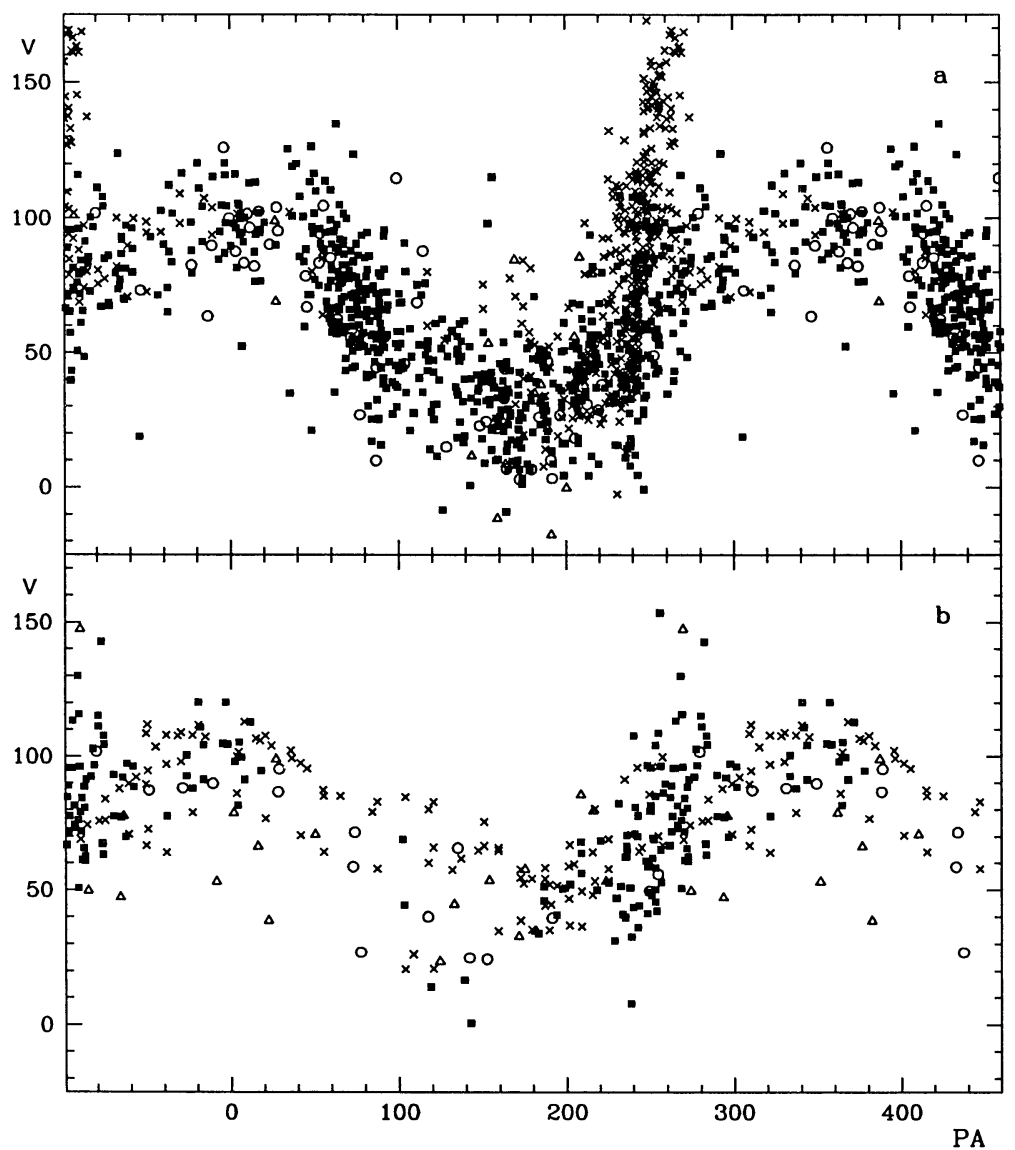

Figure 2. Radial velocities as a function of the deprojected position angles. Squares: carbon stars; circles: clusters; triangles: LPVs; crosses: HI. a) is the $4^{\circ}$ to $20^{\circ}$ zone. b) is the $3^{\circ}$ to $5^{\circ}$ zone

\section{Conclusion}

We believe that certain carbon stars, in the southwest of the LMC, do not belong to the LMC but are debris left along the SMC orbit. The peculiar radial velocity of these stars made them debris candidates. The ill-defined absolute magnitude of carbon stars prevents us from establishing if these stars lie in front or behind the LMC. One should search for RR Lyrae, red clump stars or globular clusters associated with this kinematic peculiarity to map in $3 \mathrm{D}$ the suspected orbit of the SMC.

Acknowledgments. This project was financially supported, in parts (S. D.) by the Natural Sciences and Engineering Research Council of Canada. 


\section{References}

Battinelli, P., \& Demers, S. 1999, this volume

Demers, S., Grondin, L., Irwin, M.J., \& Kunkel, W.E. 1991, in The Magellanic Clouds, R. Haynes \& D. Milne, Dordrecht: Kluwer, 473

Demers, S., \& Battinelli, P. 1998, AJ, 115, 154

Grondin, L., Demers, S., \& Kunkel, W.E. 1992, AJ, 103, 1234

Hughes, S.M.G., Wood, P.R., \& Reid, N. 1991 in The Magellanic Clouds, R. Haynes \& D. Milne, Dordrecht: Kluwer, 81

Irwin, M.J., Demers, S., \& Kunkel, W.E. 1990, AJ, 99, 191

Irwin, M.J., Demers, S., \& Kunkel, W.E. 1996, BAAS, 28, 932

Kunkel, W.E., Irwin, M.J., \& Demers, S. 1997a, A\&AS, 122, 463

Kunkel, W.E., Demers, S., Irwin, M.J., \& Albert, L. 1997b, ApJ, 488, L133 